\title{
Análisis del discurso desde la perspectiva de Michel Foucault: contribuciones y posibilidades para la investigación cualitativa en Enfermería ${ }^{1}$
}

Franciele Roberta Cordeiro $^{2}$ Maria Henriqueta Luce Kruse ${ }^{3}$

Institución: Universidad Federal de Rio Grande de Sul

\author{
ENSAYO \\ COMO CITAR
}

Leiva, V. y Villalobos, B. (2014). Implementación de la bioética en la enseñanza de enfermería. Rev. Enfermería Actual en Costa Rica, 27, 1-10. DOI: http://dx.doi.org/10.15517/revenf.v0i27.16025

\section{RESUMEN}

El punto de partida del presente estudio es la complejidad de la investigación cualitativa, la cual exige diferentes formas de análisis, cuyo fin es discutir aspectos relacionados con el cuidado, en este caso el discurso propuesto por Michel Foucault y sus intersecciones con la enfermería. En relación con el autor, se seleccionaron algunos elementos de las obras La arqueología del saber y El orden del discurso, por ejemplo, tales como la noción de autor, discurso, enunciado y disciplina. El análisis enunciativo da visibilidad a las relaciones de poder / saber que constituyen sujetos de tipo particular, en vista de la racionalidad neoliberal actual. No se trata de buscar soluciones puntuales o terminar las discusiones sobre la salud, la educación, entre otros temas, sino de instigar pensamiento, descripción y discusión en torno de lo establecido. Con este tipo de análisis es posible la (de) construcción de verdades, la descripción de relaciones y la producción de otras formas de mirar los problemas de investigación.

Palabras clave: educación; enfermería; lenguaje; metodología

\footnotetext{
${ }^{1}$ Fecha de Recepción: 20 de abril del 2014

Fecha de aceptación:30 de julio del 2014

${ }^{2}$ Doutoranda Programa de Pós-Graduação em Enfermagem da Universidade Federal do Rio Grande do Sul (UFRGS). Porto Alegre, Brasil. E-mail: franciele.cordeiro@ufrgs.brArt4 Brasil.docx

${ }^{3}$ Professor Programa de Pós-Graduação em Enfermagem da Universidade Federal do Rio Grande do Sul (UFRGS). Porto Alegre, Brasil. E-mail: kruse@uol.com.br
} 


\title{
The discourse analysis from the Michel Foucault perspective: contributions and opportunities for qualitative nursing research $^{1}$
}

\author{
Franciele Roberta Cordeiro ${ }^{2}$ \\ Maria Henriqueta Luce Kruse ${ }^{3}$
}

Institution: Federal University of Rio Grande do Sul

\begin{abstract}
ESSAY
CITED

Leiva, V. y Villalobos, B. (2014). The discourse analysis from the Michel Foucault perspective: contributions and opportunities for qualitative nursing research. Rev. Enfermería Actual en Costa Rica, 27, 1-10. DOI: http://dx.doi.org/10.15517/revenf.v0i27.16025
\end{abstract}

\begin{abstract}
The starting point of this study is the complexity of qualitative research, which requires different forms of analysis, whose purpose is to discuss issues related to the care, in this case the discourse proposed by Michel Foucault and its intersections with nursing. In relation to the author, some elements of the work The Archaeology of Knowledge and The Order of Things, for example, such as the notion of authorship speech utterance and discipline were selected. The analysis gives limited visibility to relations of power / knowledge that constitute subjects of particular type, in view of the current neoliberal rationality. It is not to seek point solutions or terminate discussions on health, education, among other topics, but to instigate thought, description and discussion of the establishment. With this type of analysis is possible (de) construction of truths, describing relations and the production of other ways to look at research problems.
\end{abstract}

Key words: education; nursing: language; methodology

\footnotetext{
${ }^{1}$ Date of receipt: July 20, 2014

Date of acceptance: July 30,2014

${ }^{2}$ Doutoranda Programa de Pós-Graduação em Enfermagem da Universidade Federal do Rio Grande do Sul (UFRGS). Porto Alegre, Brasil. E-mail: franciele.cordeiro@ufrgs.br

${ }^{3}$ Professor Programa de Pós-Graduação em Enfermagem da Universidade Federal do Rio Grande do Sul (UFRGS). Porto Alegre, Brasil. E-mail: kruse@,uol.com.br
} 


\section{Revista Electrónica Enfermeria Actual en costa Rica}

\section{INTRODUCCIÓN}

"Pero, al final, ¿qué hay de tan peligroso en el hecho de que las personas hablen de que sus discursos proliferen? Al final, ¿dónde, está el peligro?”

\section{Foucault}

Escribir. Describir. Detallar. Observar. Narrar. Todas son algunas de las acciones movilizadas por los sujetos que realizan análisis discursivo desde la perspectiva de Michel Foucault. Es necesario que los sentidos estén dispuestos y atentos para captar los detalles que pueden ser manipulados en los discursos, dado que ahí se originan los peligros a los que se refiere el filósofo francés. En este ensayo, se abordarán tales "peligros" al emplear el análisis del discurso como herramienta teórica con la investigación documental, cuyo objetivo es discutir la propuesta de Foucault y sus interfaces con la enfermería.

La inserción de referencias teóricas filosóficas en la investigación cualitativa en salud ocurre desde hace bastante tiempo. La articulación entre diferentes saberes proporciona al enfermero un trabajo diferenciado, debido a que la complejidad humana está más allá de la enfermedad y el sujeto puede ser considerado como un constructo múltiple de aspectos culturales, sociales, económicos, entre otros (Trad, 2012).

El neoliberalismo, modelo económico y político vigente, presenta nuevas formas de constitución de vida, las cuales convocan a los sujetos a participar activamente de las prácticas en torno de su cuerpo (Lazzarato, 2006; Saraiva, Veiga-Neto, 2009), las cuales modifican las relaciones entre los sujetos involucrados en el escenario del cuidado, cambio que implica importantes cuestiones éticas para los profesionales de salud, como el enfermero. Desde esa perspectiva, la investigación cualitativa en la enfermería se torna aun más compleja, debido a que en los últimos años se ha concentrado en las perspectivas emancipadora y comprensiva, al buscar las verdades sobre los cuidados, los hábitos y los comportamientos de salud a partir de la lógica subjetiva, nacida de las respuestas de los sujetos (Cordeiro, 2013).

La referencia post-estructuralista propone una mirada distinta de las cuestiones relacionadas con el cuidado, puesto que cuestiona las emergencias alrededor de los cuerpos enfermos y saludables, con base en los discursos que producen los sujetos, a partir de la óptica de la historia y de la cultura; desde esa perspectiva, se procura aprender acerca de los modos en que nos sujetamos a ciertas prácticas de salud y determinados comportamientos; además, se persigue entender cómo se llega a tomar ciertas decisiones a partir de aquello que se dice y que se toma como verdad. Lo anterior posibilita la creación de otros análisis y la producción de discursos respecto del quehacer del enfermero y de los sujetos cuidados, es decir, se introduce el universo discursivo de la enfermería, por medio del lenguaje y de las relaciones cuidador-cuidado, como una manera productiva e interesante de abordar las investigaciones que constituyen el campo de conocimiento del área en cuestión. 
En este ensayo, se emplea algunos elementos de análisis enunciativo en la perspectiva de Foucault, de estos, se presenta los puntos relevantes respecto de los cuestionamientos hechos a los materiales empíricos de análisis en las investigaciones documentales en Enfermería.

Cuando se adopta la noción de discurso como herramienta de análisis y constructo teórico, es necesario mencionar el movimiento de la Giro Lingǘstico (Linguist Turn) y sus intersecciones con el pensamiento de Foucault; al buscar esos vínculos, más que contestar a los cuestionamientos que surjan, es interesante señalar cómo se configuran las tramas de poder que garantizan la legitimidad de aquello que puede ser proferido y que adquiere estatus de verdad en determinado momento histórico.

El Giro Lingüístico fue un movimiento que rompió con los modelos filosóficos y culturales que se ocupaban del sujeto para contestar a las inquietudes del pensamiento moderno. La fenomenología, así como la Psicología, especialmente con el psicoanálisis, y el marxismo creían en una transcendencia contenida en el sujeto, quien llevaría la verdad y la búsqueda de soluciones para aspectos conductuales y sociales, idea que primó a finales del siglo XIX e inicios del XX. Al respecto, autores como Jacques Derridá, Richard Rorty y Ludwing Wittgenstein discutieron acerca del carácter no-representativo del lenguaje y su función instituidora de la realidad, de modo que ya no sería tan solo una mera simbolización de objetos y cosas. Influenciados por los positivistas del Círculo de Viena, dichos teóricos intentaron estructurar el lenguaje y su forma de organización, recurriendo a los estudios de las Ciencias Humanas y vinculándolos con las relaciones entre lenguaje, pensamiento y mundo. De manera general, para esa corriente "no hay pensamiento fuera del lenguaje, es decir, lo que no puede ser dicho no puede ser pensado - o, si quisiéramos, solo puede ser pensado aquello sobre el cual tenemos algo que decir" (Veiga-Neto, Corcini, 2007, p. 23).

En relación con lo mencionado, las obras El orden del discurso y La arqueología del saber brindan un inmenso aporte en cuanto a la comprensión de ese nuevo modo de abordar el lenguaje: en las concepciones clásicas de la lingüística, el discurso, se toma como una unidad de la lengua compuesto por sucesivas frases; dicha propuesta se materializa en un cuerpo, por medio del habla (Charaudeau, Maingueneau, 2004), entonces, partiendo de lo expuesto, el discurso se compone y está integrado por un conjunto de textos (Charaudeau, Maingueneau, 2004). Sin embargo, a partir del Giro Lingüístico, los postestructuralistas concibieron el discurso como un receptor no solo de posiciones semánticas, sino de sentidos productivos, susceptibles de control, selección, organización y de una serie de procedimientos que actúan para su interdicción o legitimación (Foucault, 2003; Charaudeau, Maingueneau, 2004).

\section{DESARROLLO}

A partir de que "¿no importa quién habla?” (Foucault, 2000, p. 264), se apartó el sujeto, se suprimió la necesidad de un ser, un autor para la enunciación del discurso, aunque no se da desde una perspectiva en el sentido de la materialidad, dado que son necesarios un cuerpo, una boca, ojos y manos para emitir lo que se 
pronuncia y que se concreta en cuanto discurso. Ahora, no interesa quien habla, mas sí "las posiciones que se ocupa en los discursos" (Fischer, 2001, p. 207).

En esa dispersión que se torna el discurso, el deseo de verdad que lo atraviesa se toma como un elemento que da visibilidad a los acontecimientos en una época. Los libros, las tecnologías, las relaciones entre los sujetos y las posiciones que ocupan socialmente dan legitimidad para lo que se enuncia; a ese poder para legitimar Foucault (2003) lo denomina como sistemas de exclusión del discurso, los cuales se tratan de procedimientos internos y externos ligados a las relaciones de poder y de los deseos de cada sujeto (Ferreira, Traversini, 2013)

En los análisis documentales en enfermería, operar con esos conceptos posibilita el entrar en los juegos de verdad que son producidos en torno a las prácticas de salud, ya sean en relación con los procesos vitales o acerca del proceso de muerte y morir. Más allá de eso, es posible señalar las relaciones entre quienes hablan y quienes escuchan; aquellos autorizados a prescribir comportamientos y que auxiliarán la modificación de las conductas en la población en cuanto al proceso salud/enfermedad.

En la relación entre los textos analizados se constata la relevancia de la investigación documental y cuán importantes son las fuentes como entrevistas, manuscritos, entre otros, para brindar información tan rica como la que se obtiene en entrevistas, grupos focales y cuestionarios, para las investigaciones cualitativas en enfermería. Por medio de los documentos, es posible reconocer aquello acerca de lo que los sujetos hablan que, al final son esos escritos y las manifestaciones orales que, en conjunto, van produciendo ciertas creencias, valores, verdades, comportamientos y prácticas respecto de los cuidados de la salud, una de las tantas posibilidades que surgen de la labor con los archivos, los cuales, tras ser comentados y relacionados con los elementos históricos de los que nacieron, apuntan el cómo se construye todo lo que nos rodea.

Entre los procedimientos de interdicción del discurso se destaca la noción de autor, quien es no solo el que enuncia algo, sino que es un "principio de agrupamiento del discurso, como unidad y origen de sus significaciones, como foco de su coherencia" (Foucault, 2003, p. 26); en este caso, la relevancia dada a un nombre, aquel que escribe, se modifica a lo largo de la historia. Durante la Edad Media, la autoría de un texto era de suma importancia, debido al control sobre los sujetos en aquel momento, puesto que era necesario conocer quién escribía algo para castigarlo o no, según lo tolerable: la autoría era un indicio de verdad. En el Renacimiento y con el inicio de la Edad Moderna, el sujeto pasa a ser menos importante frente a la capacidad de reproducción de un experimento, de una técnica o algo del género, en aquel entonces, resultaba más interesante comprobar la veracidad de algo que saber quién lo elaboró (Foucault, 2003).

En la actualidad, el lenguaje es emergente, es miramos instituidor de cuanto nos rodea y libre del sujeto transcendental. En ese sentido, la escritura puede ser considerada neutra, es decir, "un compuesto oblicuo hacia donde huye nuestro sujeto, el negro y blanco en dónde se viene a perder toda la identidad, empezando precisamente por el cuerpo que escribe" (Barthes, 2004, p. 13). En los análisis de las investigaciones documentales en enfermería no importa tanto atentar contra la singularidad de los sujetos estudiados, que nos 
remite a aquello que es dicho y que indica un conjunto de discursos, comportamientos y hábitos insertos en una cultura, los cuales van modulando las prácticas de los sujetos con sus cuerpos; mirar hacia esas prácticas invita a pensar acerca de las demandas históricas y los valores sobre los más distintos aspectos de la naturaleza humana, que circulan en cada periodo histórico y que llevan los sujetos a gobernar determinadas formas sus vidas.

Otro aspecto relevante que limita el discurso es la disciplina que se sostiene no de un conglomerado de dichos, de reglas y verdades, sino de su radicalidad, en un sistema de similares, por medio de la disposición de una serie de técnicas y estrategias aceptadas en un momento histórico (Foucault, 2003).

Un discurso inserto en una disciplina está directamente ligado a un horizonte teórico y a juegos con intereses que aseguren su circulación y que permitan su legitimación vinculada a un campo del saber. Así, la verdad contenida en una proposición, sustentada por el estatus de aquel que habla desde un lugar de poder, posibilita ir más allá de la disciplina en determinados discursos y viceversa. Así, "la disciplina es un principio que controla la producción del discurso, fija los límites por el juego de una identidad que tiene la forma de una reactualización permanente de reglas" (Foucault, 2003, p. 36). Por ejemplo, una disciplina respecto de los cuidados en el final de la vida, como los cuidados paliativos, delimitará aquello que puede ser enunciado acerca de la muerte y el morir en determinado momento. Aquello que se habla hoy en cuanto a los cuidados a la hora de la muerte, tanto en las investigaciones como en la asistencia en salud, respeta lo que fue instituido como cuerpo de conocimiento, restricto y legitimado por aquellos autorizados a hablar y a practicar cuidados del final de la vida, mediante normas establecidas por los cuidados paliativos.

Otra forma de limitar los discursos es la rarefacción, la cual selecciona y define a quienes establecen las condiciones para entrar y proferir un discurso. Acerca de esa limitante, Foucault indica que,

nadie entrará en el orden del discurso si no satisficiera a ciertas exigencias o si no fuera, de inicio, calificado para hacerlo. Más precisamente: ni todas las regiones del discurso son igualmente abiertas y penetrables; algunas son altamente prohibidas (diferenciadas y diferenciadoras), mientras otras parecen casi abiertas a todos los vientos y puestas, sin restricción previa, a la disposición de cada sujeto que habla (2003, p. 37).

Por medio de las articulaciones y la preparación de aquellos que hablan, es posible restringir la palabra y el poder conferido a los discursos. Una de las formas de esa restricción se da mediante la ritualización de la palabra (Ferreira, Traversini, 2013), proceso que se da por medio de los juegos y de las tramas de quien habla, el modo como se cuestiona, el tono de su voz, sus gestos, comportamientos, y la coyuntura personal del orador. Como ejemplo están los reportajes acerca del final de la vida en las revistas Veja y Época (revistas brasileñas), las cuales fueron analizadas en el estudio de Cordeiro (2013), quien explica que al abordar el tema del final de la vida y las prácticas con los cuerpos de los moribundos en dichas revistas, aparecen médicos autorizados a hablar y a prescribir conductas para el planeamiento de una "buena muerte". Los profesionales consultados utilizan lenguaje específico, objetos y vestimentas en común, como el estetoscopio y la gabacha, implementos que les otorgan 
autoridad para enunciar el discurso científico, de modo que la palabra es trabajada para que dos discursos alcancen un mayor número de sujetos y se produzca el efecto deseado.

La coyuntura de aquello que es dicho es favorecida por las contingencias de una época con las que se captura la rareza, así como los enunciados que se configura en torno de las prácticas en educación, en la salud, en la economía, entre otros campos. En ese sentido, es imperante la comprensión del concepto de enunciado desde dicha perspectiva filosófica, puesto que es esencial en el análisis discursivo, máxime que por medio de la relación entre los enunciados, es posible señalar y analizar el modo como la cultura y el discurso modulan las acciones de los sujetos, conforme las demandas de cada época, considerando los factores económicos, políticos, morales entre otros.

En cuanto al concepto de enunciado, para Foucault (2008) está más allá de las prácticas de enunciación, de los actos del habla, de las frases, pues, "una misma oración, con el mismo sentido, puede representar distintos enunciados" (Dreyfus y Rabinow, 2010, p. 58);no puede ser una frase por sí, puesto que las estructuras gramaticales no se reducen a los juegos del sintaxis, aunque tampoco puede ser considerada una enunciación, porque se limitaría su función al acto de hablar o de pronunciar algo. Entonces, desde esa perspectiva teórica, enunciado remite a escarbar en la historia, adentrando, desmontando y remontando acontecimientos que, no estando en una linealidad, se dispersaron, y confluye y posibilitan ciertas prácticas en torno de los cuerpos (Dreyfus y Rabinow, 2010).

Foucault no está interesado en los análisis de los actos cotidianos en torno del discurso, como ocurre en otras vertientes analíticas, como en el análisis de la conversación etnometodológica (Garcez, 2008), sino en partir de un a priori histórico, de las condiciones de emergencia y de las relaciones de poder y de saber establecidas con las que un algo puede ser discursivo en una época y para luego tornarse en enunciado.

Entretanto, no se puede negar la función de la lengua en cuanto a la existencia del enunciado, aunque lo contrario también es válido, puesto que,

si no hubiera enunciados, la lengua no existiría, pero ningún enunciado es indispensable para la existencia de la lengua. La lengua solo existe a título de sistema de construcción para enunciados posibles; pero, por otro lado, ella solo existe a título de descripción obtenida a partir de un conjunto de enunciados reales (Foucault, 2008, p. 96).

Para comprender la formación de un enunciado es necesario prestar atención al modo en el que ocurre, así como cuestionar el estatus de quien habla, los lugares desde los que emiten los discursos y las posiciones de los sujetos que hablan (Foucault, 2008).

Tal como se mencionó, el estatus que un sujeto ocupa en la sociedad asegura o no el derecho de hablar acerca de un determinado asunto, y legitima su poder de intervención y decisión, los cuales son reconocidos y operan en el funcionamiento de la coyuntura social. Las instituciones desde donde se habla aseguran que el 
discurso sea emitido con cierta frecuencia y corroborado por otros, de modo que permita el reconocimiento, la observación y la intervención, a partir de la jerarquía que posibilita la circulación e institución de verdades sobre las más variadas prácticas (Foucault, 2008). Cuando se trata de la formación de los enunciados, no se aborda al sujeto que piensa y conoce sobre aquello de lo que se habla, pero se desmonta las estructuras que provocan la ruptura, la discontinuidad y la dispersión del sujeto, que resultan en el reconocimiento de que "no es siquiera por el recurso a un sujeto transcendental, tampoco por el recurso a una subjetividad psicológica que se debe definir el régimen de sus enunciaciones" (Foucault, 2008, p. 61).

Al evocar la función histórica, relacional y dispersiva del enunciado, describiendo aquello de lo que no se trata, se puede pensar que,

el enunciado no es, pues, una estructura; es una función de existencia que pertenece, exclusivamente, a los signos, y a partir de la cual se puede decidir, en seguida, por el análisis o por la intuición, si ellos "hacen sentido" o no, segundo que regla se suceden o se superponen, de que son signos, y de que especie de acto se encuentra realizado por su formulación (Foucault, 2008, p. 63).

Cabe recordar que el analista del discurso no pretende encontrar la realidad, mucho menos descubrir lo que hizo posible que tal evento ocurriera; no se restringe a los aspectos sociales o económicos, sino que parte del principio de que la coyuntura de las circunstancias, y no los hechos aislados, posibilitará que un enunciado pueda ser formado. Por tanto, es necesaria la descripción minuciosa de los hechos, los detalles, para que sea posible reconocer cómo un objeto discursivo, como la muerte, por ejemplo, ha puesto en duda -en un momento dado- qué leyes pudieron regir su aparecimiento, qué interdicciones y limitaciones fueron puestas en juego para que se suspendiera algunas prácticas y otras no.

Foucault propone que observemos algunos puntos al analizar la formación de los discursos, tales como las condiciones de aparecimiento de un objeto de discurso, las relaciones entre instituciones y relaciones discursivas, que determinan el aparecimiento de campos de saber y de intervenciones directamente ligadas al poder sobre los cuerpos, a fin de auxiliar en la conducción y formación de los sujetos (Foucault, 2008).

\section{CONCLUSIONES}

El análisis del discurso de inspiración foucaultiana permite describir un conjunto de relaciones posibles dentro del contexto de la enfermería, la cual contribuye al entendimiento acerca de la producción del cuidado, de la salud y sobre los modos por los cuales los individuos se sujetan a los discursos que circulan, mediante los que adoptan ciertas decisiones respecto de su cuerpo, de su vida y de su muerte.

En ese juego, es necesario detallar las relaciones de saber/poder que circunscriben las prácticas institucionales y que son intrínsecas a la producción del sujeto y de su subjetividad. Para conocer esas constituciones del sujeto, de las posiciones que él es llamado a ocupar o también de la constitución de un objeto 
discursivo en una época, es necesario realizar una descripción minuciosa de las tácticas, estrategias y procedimientos utilizados para conducir a los sujetos, sean ellos dispuestos por el Estado, por los profesionales de salud o cualquier institución (Paraíso y Meyer, 2012).

Tras mencionar lo anterior, se corrobora que el análisis es una herramienta fundamental para trabajar los discursos emergentes en las investigaciones en Enfermería; a partir de las nociones de autor/autoría, disciplina y enunciado, procedimientos que impiden o permiten la circulación de ciertas verdades en los discursos de los enfermeros, es posible analizar los problemas bajo otros ángulos, discutiendo las prácticas de cuidado a partir de la cultura, del lenguaje y de las condiciones históricas que permiten que algo sea inventado en este tiempo. La idea es pensar de otro modo las cuestiones de investigaciones y los hallazgos en Enfermería, ampliando los cuestionamientos, fomentando los debates y construyendo otras verdades que producen el conocimiento y el cuidado en esa área.

\section{REFERENCIAS BIBLIOGRAFICAS}

Barthes, R. (2004). O rumor da língua. São Paulo: Martins Fontes.

Charaudeau P., Maingueneau, D. (2004). Dicionário de Análisis do Discurso. São Paulo: Contexto.

Cordeiro, F. (2013). Eu decido meu fim? A mídia e a produción de sujetos que governam sua morte. Porto Alegre: Programa de Pósgrado en Enfermería da Universidade Federal do Rio Grande do Sul.

Dreyfus H., Rabinow P. (2010). Michel Foucault, uma trajetória filosófica. Para além do estruturalismo e da hermenêutica. Rio de Janeiro: Forense Universitária.

Ferreira, M., Traversini, C (2013). A análise foucaultiana de discurso como ferramenta metodológica de pesquisa. Educación \& Realidade, v. 38, n. 1, pág. 207-226.

Fischer, R. (2001). Foucault e a análise do discurso em educação. Cadernos de Pesquisa. Fundação Carlos Chagas, $n^{\circ} 114$, pág. 197-223. Recuperado em 28 de janeiro de 2014: http://www.scielo.br/pdf/cp/n114/a09n114.pdf

Foucault, M. (2003). A ordem do discurso: aula inaugural no Collège de France, pronunciada em 2 de dezembro de 1970. 9 ed. São Paulo: Loyola.

Foucault, M. (2000). O que é um autor? In: Ditos e Escritos III- Estética: literatura e pintura, música e cinema. Rio de Janeiro: Forense Universitária.

Foucault, M. (2008). A arqueologia do saber. 7 ed. Rio de Janeiro: Forense Universitária. 
Garcez, P. (2008). A perspectiva da Análisis da Conversa Etnometodológica sobre o uso da linguagem em interação social. In: Fala-em-interación social: uma introdução à Análise da Conversa Etnometodológica. Londer LL, Jung N (Orgs.). Campinas: Mercado de Letras.

Lazzarato, M. (2006). As revolucçõesiones do capitalismo. Rio de Janeiro: Editora Record.

Paraíso, M. (2012). Metodologias de pesquisas pós-críticas em educação e currículo: trajetórias, pressupostos, procedimentos e estratégias analíticas. In: Metodologias de pesquisas pós-críticas em educação. Meyer DE, Paraíso MA (Orgs.). Belo Horizonte: Mazza Edições.

Saraiva, K., Veiga, A. (2009). Modernidade líquida, capitalismo cognitivo e educação contemporânea. Educação \& Realidade, v. 34, n. 2, 187-201.

Trad, L.A.B. (2012). Trabalho de campo, narrativa e produción de conhecimento na pesquisa etnográfica contemporânea: subsídios ao campo da saúde. Ciência \& Saúde Coletiva, nº 13, v. 2, 627-633. Recuperado em 27 de janeiro de 2014 em: http://www.scielo.br/pdf/csc/v17n3/v17n3a08

Veiga, A., Corcini, M. (2007). Identidade, cultura e semelhança de família: as contribuições da virada linguística. In: Bizarro, R (Org.). Eu e os outros: estudos multidisciplinares sobre identidade(s), diversidade(s) e práticas culturais. Porto: Areal. 